\title{
FGF23 gene regulation by 1,25-dihydroxyvitamin D: opposing effects in adipocytes and osteocytes
}

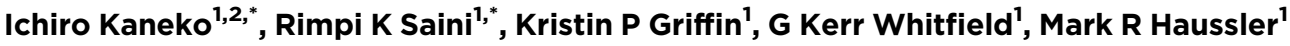 \\ and Peter W Jurutka ${ }^{1,2}$
}

${ }^{1}$ Department of Basic Medical Sciences, University of Arizona College of Medicine, 425 N. Fifth Street, Phoenix, Arizona 85004, USA

${ }^{2}$ School of Mathematical and Natural Sciences, Arizona State University, 4701 W. Thunderbird Road, Phoenix, Arizona 85306, USA

*(I Kaneko and R K Saini contributed equally to this work and are therefore to be considered co-first authors)
Correspondence should be addressed to P W Jurutka Email pjurutka@asu.edu

\begin{abstract}
In a closed endocrine loop, 1,25-dihydroxyvitamin $D_{3}(1,25 \mathrm{D})$ induces the expression of fibroblast growth factor 23 (FGF23) in bone, with the phosphaturic peptide in turn acting at kidney to feedback repress CYP27B1 and induce CYP24A1 to limit the levels of 1,25D. In 3T3-L1 differentiated adipocytes, 1,25D represses FGF23 and leptin expression and induces $C / E B P \beta$, but does not affect leptin receptor transcription. Conversely, in UMR-106 osteoblast-like cells, FGF23 mRNA concentrations are upregulated by 1,25D, an effect that is blunted by lysophosphatidic acid, a cell-surface acting ligand. Progressive truncation of the mouse FGF23 proximal promoter linked in luciferase reporter constructs reveals a 1,25D-responsive region between -400 and $-200 \mathrm{bp}$. A $0.6 \mathrm{~kb}$ fragment of the mouse FGF23 promoter, linked in a reporter construct, responds to 1,25D with a fourfold enhancement of transcription in transfected $\mathrm{K} 562$ cells. Mutation of either an ETS1 site at $-346 \mathrm{bp}$, or an adjacent candidate vitamin D receptor (VDR)/Nurr1-element, in the $0.6 \mathrm{~kb}$ reporter construct reduces the transcriptional activity elicited by $1,25 \mathrm{D}$ to a level that is not significantly different from a minimal promoter. This composite ETS1-VDR/Nurr1 cis-element may function as a switch between induction (osteocytes) and repression (adipocytes) of FGF23, depending on the cellular setting of transcription factors. Moreover, experiments demonstrate that a $1 \mathrm{~kb}$ mouse FGF23 promoter-reporter construct, transfected into MC3T3-E1 osteoblast-like cells, responds to a high calcium challenge with a statistically significant 1.7- to 2.0-fold enhancement of transcription. Thus, the FGF23 proximal promoter harbors cis elements that drive responsiveness to 1,25D and calcium, agents that induce FGF23 to curtail the pathologic consequences of their excess.
\end{abstract}
Key Words
- gene regulation
- hormone receptors
- adipose
- transcription factors
- vitamin D

Journal of Endocrinology (2015) 226, 155-166

\section{Introduction}

The vitamin $\mathrm{D}$ receptor (VDR) regulates transcription in response to its 1,25-dihydroxyvitamin $\mathrm{D}_{3}(1,25 \mathrm{D})$ ligand by forming a heterodimer with one of the retinoid $\mathrm{X}$ receptors (RXRs) and binding to vitamin $\mathrm{D}$ responsive elements (VDREs) near or remote to each target gene (Haussler et al. 2013). Fibroblast growth factor 23 (FGF23) http://joe.endocrinology-journals.org DOI: $10.1530 / J O E-15-0225$
(C) 2015 Society for Endocrinology Printed in Great Britain
Published by Bioscientifica Ltd. 
is an osteoblast/osteocyte-elaborated, phosphaturic hormone that feedback represses CYP27B1 and induces CYP24A1 to limit the levels of active 1,25D (Haussler et al. 2012, Quarles 2012). FGF23 expression is governed by a complex network of hormones, growth factors, and minerals, including $1,25 \mathrm{D}$, parathyroid hormone (PTH), leptin, cortisol, FGF2, iron, calcium, and phosphate (David et al. 2013, Saini et al. 2013, Lanske \& Razzaque 2014). VDR-null mice possess vanishingly low circulating levels of FGF23 (Yu et al. 2005), indicating that the skeletal secretion of FGF23 is dependent on the presence of VDR. The precise mechanism by which $1,25 \mathrm{D}$, via the nuclear VDR, regulates FGF23 gene transcription is yet to be defined. Kolek et al. (2005) originally reported that in osteocyte-like UMR-106 cells, FGF23 mRNA levels are dramatically upregulated by $1,25 \mathrm{D}$. This effect was reproduced, in vivo, with 1,25D-injected mice displaying increased FGF23 mRNA in tibia and calvaria, accompanied by a striking enhancement in circulating immunoreactive FGF23 protein (Kolek et al. 2005). Induction of FGF23 by $1,25 \mathrm{D}$ in UMR-106 cells is sensitive to inhibition by cycloheximide (Kolek et al. 2005, Haussler et al. 2010), suggesting that the transcriptional effect may be secondary and dependent on the induction of an intermediary transcription factor. However, the time course of FGF23 mRNA upregulation by 1,25D in UMR106 cells, with the first observable effect at $2 \mathrm{~h}$ and the peak effect at $12 \mathrm{~h}$ post $1,25 \mathrm{D}$ treatment, is essentially identical to that of osteopontin mRNA induction by $1,25 \mathrm{D}$ (R K Saini, unpublished). This observation is puzzling because the action of $1,25 \mathrm{D}$ on osteopontin transcription is insensitive to cycloheximide (Haussler et al. 2010), rendering it a definitive primary effect that occurs in temporal concert with the apparently more complex induction of FGF23.

Employing ROS 17/2.8 osteoblast-like cells, Liu et al. (2006) independently confirmed the findings of Kolek et al. (2005), reporting a substantial induction of FGF23 by $1,25 \mathrm{D}$ that was prevented by cotransfection with a dominant negative VDR plasmid (Jurutka et al. 1997). They also identified a candidate VDRE in the form of a direct repeat with a spacer of three nucleotides (DR3) VDRE, AGGTTActgAGTTCC, located at -1124 bp in relation to the transcription start site in the mouse FGF23 gene (Liu et al. 2006). Importantly, site-directed mutagenesis of this VDRE in the context of a mouse FGF23 promoter-reporter construct compromised the ability of 1,25D to stimulate transcription (Liu et al. 2006). The results of Liu et al. (2006) provide evidence that 1,25D induces FGF23 in a primary fashion, mediated by VDR binding to a VDRE near the proximal promoter of the mouse FGF23 gene. However, it has been reported that the FGF23 gene region in mouse osteocytes is not marked by detectable 1,25D-dependent VDR/RXR binding sites when ChIP-Seq analysis is carried out (St John et al. 2014). Therefore, the in vivo relevance of VDREs residing in the mouse FGF23 gene remains to be confirmed.

Recently, functional VDREs have been identified in the human FGF23 gene at $-35.7 \mathrm{~kb}$ (GGGAGAatgAGGGCA), at $-16.2 \mathrm{~kb}$ (TAACCCtgctttAGTTCA, an everted repeat with a spacer of six nucleotides), and at $+8.6 \mathrm{~kb}$ (AGGGCAggaAGGACA) in relation to the transcription start site (Saini et al. 2013). Notably, these human VDREs are located at some distance from the promoter, but each occurs in a cluster of binding sites for C/EBP and RUNX2 (Saini et al. 2013), indicating they may lie in cis-regulatory modules (CRMs) for control by the vitamin D hormone of osteoblast-expressed genes (Meyer et al. 2010). Based on the observation that FGF23 induction by $1,25 \mathrm{D}$ is at least partially cycloheximide sensitive (Haussler et al. 2010) and the fact that 1,25D upregulates ETS1, a transcription factor that cooperates with VDR (Dittmer 2003), we (Saini et al. 2013) previously concluded that $1,25 \mathrm{D}$ induces human FGF23 production directly (primarily) via multiple VDREs and indirectly (secondarily) via stimulation of ETS1 expression, with VDR and ETS1 cooperating in the induction of FGF23 through DNA looping and the generation of euchromatin architecture (Saini et al. 2013).

The present communication reports that $1,25 \mathrm{D}$ represses FGF23 expression in adipocytes, contrasting with the induction of FGF23 by 1,25D in osteocytes. Thus, the directionality of FGF23 regulation by $1,25 \mathrm{D}$ is cell selective. Evidence also is provided for an ETS1-VDRE/Nurr1 composite element that is conserved across species in the proximal promoter of the FGF23 gene. Combined with our previous report that 1,25D induces ETS1 (Saini et al. 2013), we conclude that this ETS1-VDRE/Nurr1 composite element may play a central role in a combined primary and secondary regulation of FGF23 mRNA by the vitamin D hormone.

\section{Materials and methods}

\section{Cell culture}

Five mammalian cell lines (from the American Type Culture Collection, Manassas, VA, USA) were employed in this study. Rat osteoblast/osteocyte-like (UMR-106) cells were cultured in DMEM/F12 (Hyclone Laboratories,

Published by Bioscientifica Ltd. 
Logan, UT, USA) supplemented with $2.5 \mathrm{mM}$ L-glutamine, and human embryonic kidney 293 (HEK293) cells were grown also in DMEM (Hyclone). Mouse embryonic preadipose (3T3-L1), preosteoblast (3T3-E1), and human K562 myelogenous leukemia cells were cultured with DMEM/high glucose (Hyclone). All cells were passaged in the above indicated media supplemented with $10 \%$ fetal bovine serum (FBS) and penicillin/streptomycin under a humidified atmosphere of $5 \% \mathrm{CO}_{2}$ in air at $37{ }^{\circ} \mathrm{C}$. $\mathrm{K} 562$ cells were maintained as suspensions but received treatments identical to those of adherent cultures. Mouse 3T3L1 cells were differentiated to adipocytes as follows: attached cells were incubated with Differentiation Medium A (DMEM/high glucose/10\% FBS/penicillin/ streptomycin $+1 \mu \mathrm{g} / \mathrm{ml}$ insulin $+0.25 \mu \mathrm{M}$ dexamethasone $+0.5 \mathrm{mM}$ isobutylmethylxanthine) for 3 days, followed by 4 days (with medium change after the first 2 days) in Differentiation Medium B (DMEM/high glucose/10\% FBS-penicillin/streptomycin $+1 \mu \mathrm{g} / \mathrm{ml}$ insulin). On day 7 , cells were treated with hormone or ethanol vehicle, followed by harvest on day 8 .

\section{Oil Red O staining}

3T3-L1 cells or adipocytes were washed twice with PBS, fixed in 10\% formalin for $10 \mathrm{~min}$ at RT and washed again twice with PBS. Cells were then stained with a filtered Oil Red O solution (in 60\% isopropanol) for
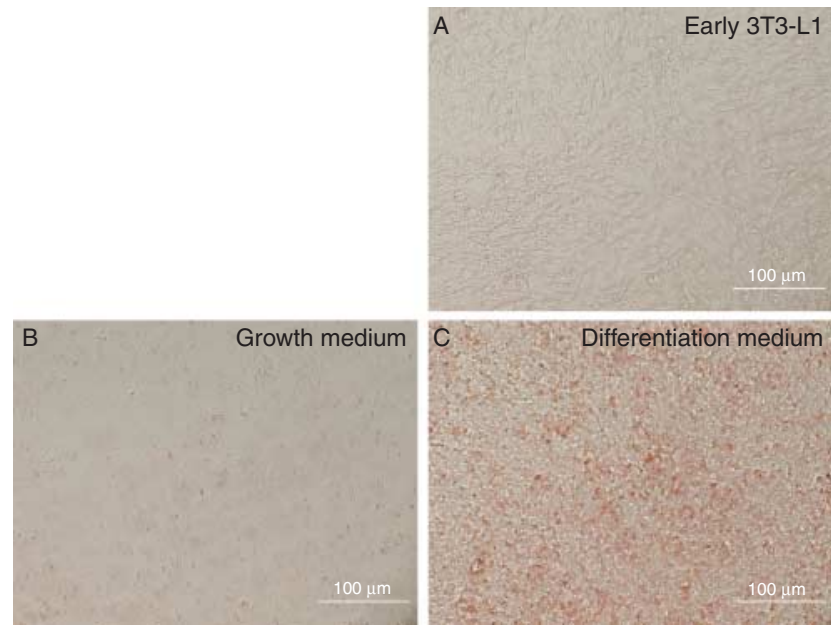

\section{Figure 1}

Oil Red O staining shows differentiation of MC3T3-L1 cells into adipocytes. (A) Undifferentiated 3T3-L1 cells cultured in standard medium. (B) 3T3-L1 cells maintained in growth medium. (C) 3T3-L1 cells cultured in differentiation medium for 7 days and stained with Oil Red $O$ as described in 'Materials and methods'. A full colour version of this figure is available at http://dx.doi.org/10.1530/JOE-15-0225.
30 min at RT. Cells were washed twice with PBS and visualized (Fig. 1).

\section{Plasmid constructs}

The $1.0 \mathrm{~kb}$ mouse FGF23 promoter and truncations thereof $(0.6,0.4,0.2$, and $0.06 \mathrm{~kb})$ in the reporter construct pGL3-basic were a kind gift from Drs Mikiko Ito and Kenichi Miyamoto, Tokushima University, Tokushima City, Japan (Ito et al. 2005). Mutations of the VDRE and ETS1 binding sites in the $0.6 \mathrm{~kb}$ mouse FGF23 promoter construct were carried out using the following oligonucleotides to introduce the mutations: to mutate the ETS1 site, 5'-gtactgctggctgccttcacaCTAAATgatggaagtggggac-3' (ETS1 site capitalized, mutated bases underlined); to mutate the VDRE/Nurr1 site, 5' -cctgatggaAGTGGGgacTTGTCAacaaatgacccagg-3' (VDRE/Nurr1 site capitalized, mutated bases underlined). To create single mutations, the indicated oligonucleotide and its complement were utilized along with the $0.6 \mathrm{~kb}$ mouse FGF23 reporter construct and a Quick Change II XL Kit (Agilent Technologies, Santa Clara, CA, USA) according to the manufacturer's instructions. To construct the double mutation, the MutETS1 plasmid was further mutagenized using the VDRE oligonucleotide pair described above. Successful mutation in each case was verified by DNA sequencing of the MutETS1, MutVDRE, and double $(2 \times$ Mut $)$ plasmids.

\section{Transfection and dual luciferase reporter assay}

Transfection of K562 cells in suspension was performed by plating $2 \times 10^{5}$ cells/well in 24 -well plates, then using PolyJet reagent (SignaGen Laboratories, Gaithersburg, MD, USA) according to the manufacturer's instructions. Briefly, each well received $2.0 \mu$ PolyJet reagent, $500 \mathrm{ng}$ of the mouse FGF23 promoter constructs to be tested, $100 \mathrm{ng}$ of pSG5-hVDR (plasmid expressing human VDR), and $20 \mathrm{ng}$ of pRL-null (Renilla luciferase reporter). After transfection, each well was treated with either 1,25D (final concentrations of $10 \mathrm{nM}$ ) or an ethanol vehicle for $20 \mathrm{~h}$ at $37^{\circ} \mathrm{C}$. Phosphate- or calcium-treated cells were treated with the appropriate dilution of $1 \mathrm{M}$ sodium phosphate or $4.2 \mathrm{M} \mathrm{CaCl}_{2}$ in water. After incubation, cells were washed with PBS and lysed in $1 \times$ passive lysis buffer (Promega). Lysates were harvested and analyzed sequentially for Firefly luciferase and Renilla luciferase activity using a Dual Luciferase Assay Kit (Promega Corp., Madison, WI, USA) and a Sirius Luminometer (Zylux Corp., Huntsville, AL, USA) according to the manufacturers' protocols.

Published by Bioscientifica Ltd 


\section{Quantitative real-time PCR}

HEK293 cells were plated at $1 \times 10^{6}$ cells in a $60 \mathrm{~mm}$ dish. UMR-106 cells were plated at $5 \times 10^{5}$ cells/well in a six-well plate, allowed to attach overnight, then treated with lysophosphatidic acid (LPA) and analogues, $1,25 \mathrm{D}$, or ethanol vehicle in media containing $1 \%$ charcoal/dextran stripped FBS for $24 \mathrm{~h}$. LPA and Ki16425 (Cayman Chemical Co., Ann Arbor, MI, USA) and OMPT (Avanti Polar Lipids, Alabaster, AL, USA) were dissolved in ethanol and used as described in the figure legends.

Cells were harvested and total cellular RNA was isolated utilizing an Aurum Total RNA Mini Kit (Bio-Rad). The RNA obtained was quantified using A260/280 spectrophotometry. DNase-treated RNA $(1 \mu \mathrm{g})$ was reverse transcribed using the iScript cDNA Synthesis Kit (Bio-Rad). The obtained cDNA was used in $20 \mu \mathrm{l}$ PCRs containing $10 \mu \mathrm{l}$ FastStart Universal SYBR Green Master (Roche) and primers. Reactions were performed in 96-well PCR plates on an 7500 Fast Instrument (Applied Biosystems, Waltham, MA, USA). Data were analyzed using the comparative $\mathrm{Ct}$ method as the means of relative quantitation, normalized to an endogenous reference $(\mathrm{GAPDH})$ and relative to a calibrator (normalized $\mathrm{Ct}$ value from vehicle-treated cells), and expressed as $2^{-\Delta \Delta C t}$ according to Applied Biosystems' user bulletin 2: Rev B, 'relative quantitation of gene expression'. Primer sets for real-time PCR were as follows: rat FGF23: forward, 5'-ACGGAACACCCCATCAGACTATC- $3^{\prime}$ and reverse, 5'-TATCACTACGGAGCCAGCATCCTC-3'; rat CYP24A1: forward, 5'-GATCACCTTTCCAAGAAGGAACT- $3^{\prime}$ and reverse, $5^{\prime}$-AGAGAATCCACATCAAGCTGTTC- ${ }^{\prime}$; rat GAPDH: forward, 5'-AGGTCGGTGTGAACGGATTTG-3' $3^{\prime}$ and reverse, $5^{\prime}$-CATTCTCAGCCTTGACTGTGCC-3'; human GAPDH: forward, $5^{\prime}$-ACAACTTTGGTATCGTGGAAGGAC-3' and reverse, 5'-CAGGGATGATGTTCTGGAGAG-3' or forward, 5'-TGACAACTTTGGTATCGTGGAAGG-3' and reverse, 5'-AGGGATGATGTTCTGGAGAGCC-3'; mouse GAPDH: forward, $5^{\prime}$-TTCCGTGTTCCTACCCCCAATG-3' and reverse, 5'-TGCCTGCTTCACCACCTTCTT-3'; human CYP24A1: forward, 5'-CAGCGAACTGAACAAATGGTCG-3' and reverse, 5'-TCTCTTCTCATACAACACGAGGCAG-3'; mouse Cyp24a1: forward, 5'-CGTTCTGGGTGAATACACGCTAC- $3^{\prime}$ and reverse, 5'-TTCGGGTCTAAACTTGTCAGCATC-3'; mouse leptin: forward, 5'-GTGCCTATCCAGAAAGTCCAG-3' and reverse, 5'-TGAAGCCCAGGAATGAAGTC-3'; mouse leptin receptor (ObR): forward, 5'-GCATGCAGAATCAGTGATATTTGG-3' and reverse, 5'-CAAGCTGTATCGACACTGATTTCTTC-3'; human FGF23: forward, 5'-TGCTGGCTTTGTGGTGATTA-3' and reverse, $5^{\prime}$-TTCTCCGGGTCGAAATAGTG-3'; mouse FGF23: forward,
5'-GGTGATAACAGGAGCCATGAC-3' and reverse, 5'-TGCTTCTGCGACAAGTAGAC-3'; mouse C/EBPß: forward, 5'-AGCGGCTGCAGAAGAAGGT- ${ }^{\prime}$ and reverse, 5'-GGCAGCTGCTTGAACAAGTTC-3'; mouse PPAR $\gamma$ : forward, $5^{\prime}$-CGCTGATGCACTGCCTATGA- ${ }^{\prime}$ and reverse, 5'-AGAGGTCCACAGAGCTGATTCC-3'; mouse resistin: forward, 5'-TCAACTCCCTGTTTCCAAATGC- $3^{\prime}$ and reverse, $5^{\prime}$-TCTTCACGAATGTCCCACGA-3'; and mouse aP2: forward, 5'-CATGGCCAAGCCCAACAT- $3^{\prime}$ and reverse, 5'-CGCCCAGTTTGAAGGAAATC-3'.

\section{Statistical analysis}

Data are expressed as means \pm s.D. Statistical differences between two groups were determined by a two-sided Student's $t$-test. Differences among multiple groups were analyzed by ANOVA. A $P$ value $<0.05$ was considered significant.

\section{Results}

\section{1,25D represses the expression of FGF23 in 3T3-L1 differentiated adipocytes}

Previously, only the upregulation of FGF23 mRNA expression by $1,25 \mathrm{D}$ has been reported in mammalian cells (Haussler et al. 2012). However, as illustrated in Fig. 2, in differentiated 3T3-L1 mouse adipocytes, FGF23 is significantly and dosedependently repressed by $1,25 \mathrm{D}$. When undifferentiated 3T3-L1 cells are transferred from growth medium (GM) to differentiation medium (DM), FGF23 expression is reduced by $50 \%$, followed by a further suppression of FGF 23 mRNA by $1,25 \mathrm{D}$ that is detectable at a hormone concentration as low as $0.1 \mathrm{nM}$, but first becomes statistically significant at $1.0 \mathrm{nM}$ 1,25D (Fig. 2). The inset to Fig. 2 depicts the average of three independent experiments like that illustrated in the main body of the figure, verifying the 1,25D dose-dependency of FGF23 suppression in differentiated 3T3-L1 adipocytes.

\section{Vitamin D hormone control of other adipocyte-expressed genes}

To determine if the repression of FGF23 by $1,25 \mathrm{D}$ in adipocytes is unique, the effect of the hormone on other adipocyte-expressed genes was investigated. Initially, for reference, after 3T3-L1 cells are transferred from GM to DM, leptin is induced sixfold, leptin receptor is repressed 20-fold, and Cyp24a1 is relatively unchanged (data not shown). As shown in Fig. 3A and C, leptin expression is downregulated by $1,25 \mathrm{D}$ in 3T3-L1 differentiated adipocytes. This effect is statistically significant at $100 \mathrm{nM} 1,25 \mathrm{D}$, but because of the

Published by Bioscientifica Ltd. 


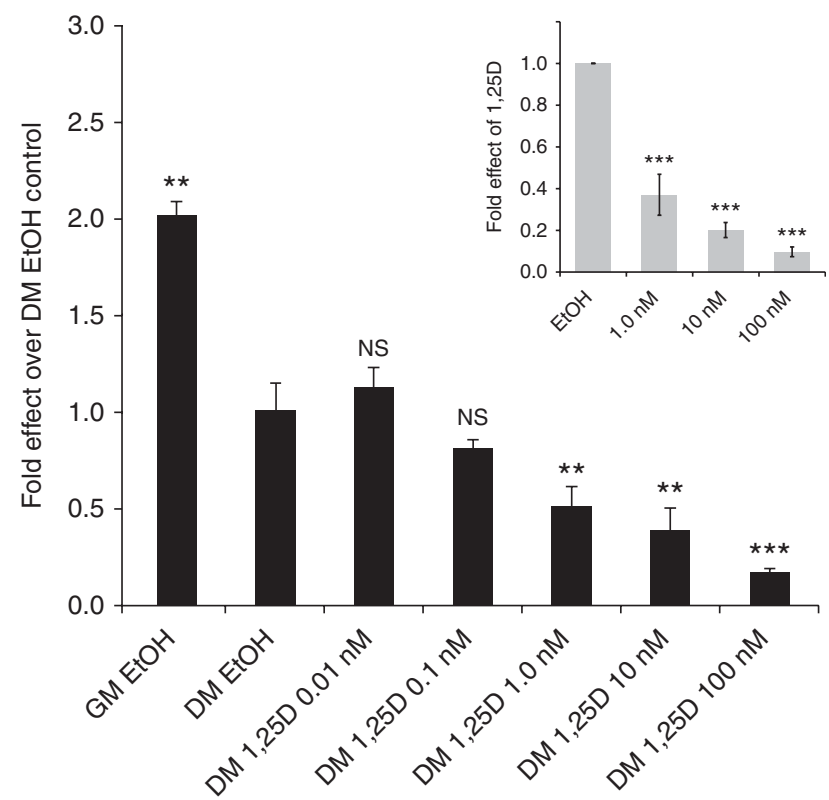

Figure 2

FGF23 mRNA repression by 1,25D in 3T3-L1 adipocytes. 3T3-L1 cells were grown as described in 'Materials and methods' and treated with 1,25D for $24 \mathrm{~h}$ at the indicated concentrations. mRNA levels for FGF23 were determined by real-time PCR. GM, growth medium; DM, differentiation medium. The inset depicts the average of three independent experiments identical to that illustrated in the main body of the figure, except that only doses of 1, 10, and $100 \mathrm{nM} \mathrm{1,25D}$ were tested. Each bar represents the average of three independent experiments performed in triplicate \pm s.D. All statistics are calculated relative to the DM EtOH set at 1.0. $* * P<0.01$ and $* * * P<0.001$.

high variability of these data, it is difficult to define dose responsiveness. Nevertheless, repression of leptin is evident and is unusually rapid in that maximal repression occurs within only $2 \mathrm{~h}$ of hormone treatment (data not shown). In contrast, leptin receptor $\mathrm{mRNA}$ is unaffected by treatment of 3T3-L1 differentiated adipocytes with 1,25D (Fig. 3B).
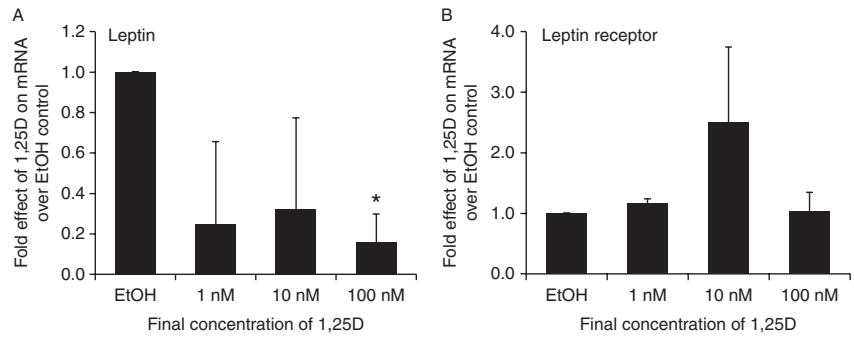

Figure 3

1,25D represses leptin mRNA while inducing Cyp24a1 in 3T3-L1 adipocytes. Differentiated 3T3-L1 adipocytes were treated with 1,25D for $24 \mathrm{~h}$ at the indicated concentrations, and mRNA levels for the indicated gene products were determined by real-time PCR (A, B and D) or by gel electrophoresis of PCR products after 40 cycles (C). Cyp24a1 is a positive control known to be
Cyp24a1 was employed as a highly induced positive control for VDR-mediated 1,25D action to ensure that the tested cells expressed VDR appropriately and responded to the $1,25 \mathrm{D}$ ligand in the expected fashion. Indeed, Fig. 3D illustrates a dramatic, dose-dependent enhancement of Cyp24a1 mRNA concentrations elicited by the vitamin D hormone (nearly 500-fold at the maximal dose of $100 \mathrm{nM}$ ), indicating that the differentiated 3T3-L1 mouse adipocyte system represents a valid model for probing VDR-targeted gene expression. Furthermore, as summarized in Fig. 4, among other genes expressed in 3T3-L1 differentiated adipocytes, $1,25 \mathrm{D}$ induces $C / E B P \beta$, whereas $P P A R \gamma$, resistin, and $a P 2$ are all regulated biphasically, with induction by $1 \mathrm{nM} \mathrm{1,25D}$ and dose-dependent repression by 10-100 nM hormone. Thus, among the genes regulated by $1,25 \mathrm{D}$ in adipocytes, FGF23 stands out as unique with respect to its unequivocal repression by $1,25 \mathrm{D}$ in a classic dose-dependent fashion. To assess the physiologic significance of FGF23 repression by 1,25D in adipose, it will ultimately be necessary to monitor FGF23 in intact mouse and human fat tissue to investigate possible in vivo target pathways affecting metabolism.

\section{1,25D induces the expression of FGF23 in UMR-106 osteoblasts; modulation by LPA}

To contrast FGF23 regulation in adipocytes vs in its skeletal endocrine source, UMR-106 osteoblast/osteocyte-like cells were treated with $1,25 \mathrm{D}$, as well as with LPA, a known potentiator of $1,25 \mathrm{D}$ action to induce osteoblast maturation and alkaline phosphatase expression (Gidley et al. 2006, Mansell \& Blackburn 2013). As illustrated in Fig. 5A, $1,25 \mathrm{D}$ dramatically (1920-fold) induces FGF23 in UMR106 cells, as previously observed in our laboratory and by others (Kolek et al. 2005, Liu et al. 2006, Bergwitz \&
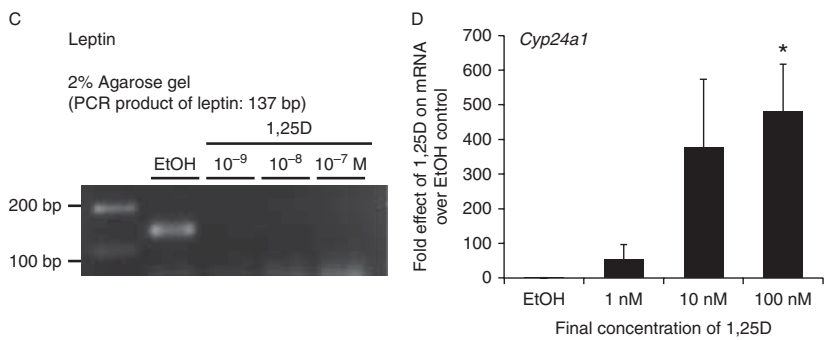

upregulated by $1,25 \mathrm{D}$. Statistical significance is depicted in the body of each panel. Each bar represents the average of three independent experiments performed in triplicate \pm s.D. *1,25D-treated groups statistically significantly different from ETOH control $(P<0.05)$.

Published by Bioscientifica Ltd. 

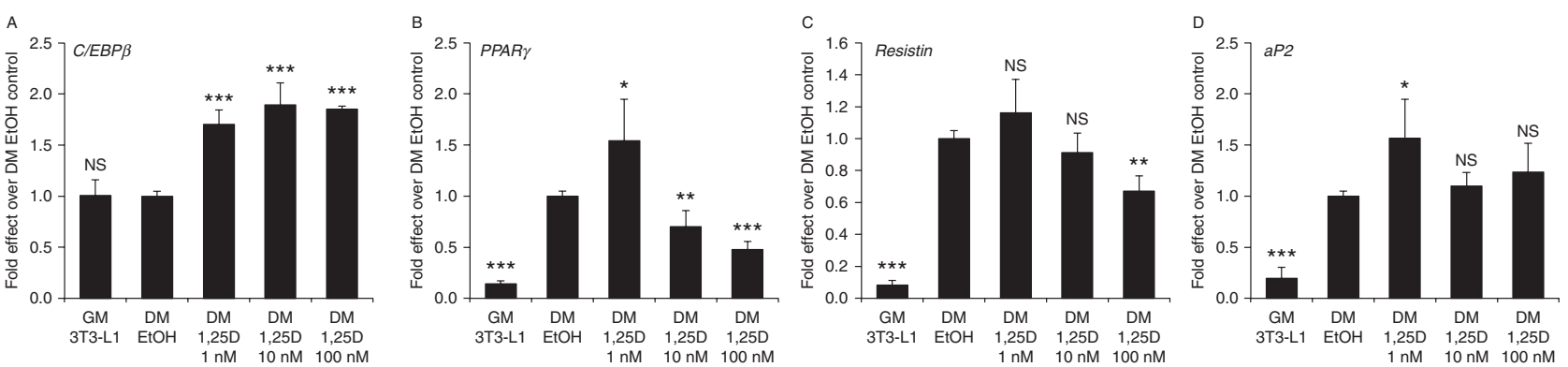

Figure 4

$1,25 D$ induces C/EBP $\beta$ mRNA and biphasically regulates $P P A R \gamma$, resistin, and aP2 in 3T3-L1 adipocytes. 3T3-L1 cells were grown as described in 'Materials and methods' and the legend to Fig. 2, then treated with 1,25D for $24 \mathrm{~h}$ at the indicated concentrations, and mRNA levels were determined by realtime PCR for the following genes: (A) CIEBP $\beta$, (B) PPAR $\gamma$, (C) resistin, and (D)

Juppner 2010). Curiously, LPA and its OMPT agonist blunt the induction of FGF23, whereas the LPA antagonist, Ki16425, slightly amplifies the upregulation of FGF23 by $1,25 \mathrm{D}$ (Fig. 5A). A very similar profile of modulation by LPA agonists/antagonists emerges (Fig. 5B) with respect to the induction of the positive control gene, Cyp24a1, by 1,25D in UMR-106 cells. In contrast, when HEK293 cells are similarly probed for $1,25 \mathrm{D}$ control of FGF23 gene expression and its modulation by LPA (Fig. 6A), the results differ markedly from those obtained in osteoblasts. Specifically, FGF23 expression is not affected by $1,25 \mathrm{D}$ in HEK293 cells (Fig. 6A), whereas LPA agonists enhance the action of $1,25 \mathrm{D}$ to induce CYP24A1 positive control mRNA (Fig. 6B). The LPA antagonist Ki16425 had no significant effect on $1,25 \mathrm{D}$ action in these experiments. Thus, LPA acts more traditionally as a $1,25 \mathrm{D}$ potentiator of CYP24A1 induction in HEK293 cells as opposed to its reverse effect in UMR-106 osteoblasts. This finding indicates that not only is FGF23 regulation by $1,25 \mathrm{D}$ cell selective in terms of its directionality but also modulation of this effect by LPA agonists/antagonists differs according to the cell setting.

\section{Dissection of the mouse FGF23 promoter by informatics, truncation, and mutagenesis}

We have reexamined the mouse FGF23 proximal promoter (DNA sequence listed in Fig. 7) for transactivator binding sites that might mediate control of FGF23 mRNA expression. As depicted in Fig. 7, we observed a collection of conserved, consensus cis elements between -110 and $-347 \mathrm{bp}$ in relation to the start of transcription. Most prominent in this collection are three ETS1, two Nurr1 (bold type), two GATA, and single AP1 and LEF1 sites. It is noteworthy that AP1 and LEF1 cis elements are often
aP2. GM, growth medium; DM, differentiation medium. Each bar represents the average of three independent experiments performed in triplicate \pm s.D. All statistics are calculated relative to the DM EtOH set at 1.0. $* P<0.05, * * P<0.01$, and $* * * P<0.001$.

associated with VDREs in 1,25D-regulated genes (Ozono et al. 1990, Luderer et al. 2011). Meir et al. (2014) recently provided evidence that PTH induces FGF23 in osteoblasts via activation of Nurr1, a transcription factor involved in the development of midbrain dopaminergic neurons (Sakurada et al. 1999). Strikingly, one of the conserved Nurr1 sites in the FGF23 promoter (Meir et al. 2014) exactly overlaps the $3^{\prime}$ putative half element in the proposed new VDRE (Fig. 7). Equally important, ETS1, which was implicated as a partner with VDR in controlling FGF23 in a previous study (Saini et al. 2013), possesses a cis-docking site in the mouse FGF23 promoter that is only 6 bp $5^{\prime}$ of a newly discovered candidate VDRE/Nurr1 (AGTGGGgacAGGTCA) site at -334 bp (highlighted in light purple in Fig. 7). This novel VDRE is distinct from the VDRE reported by Liu et al. (2006) at -1124 bp (highlighted in dark gray in Fig. 7). We hypothesized that the -110 to $-346 \mathrm{bp}$ region of the FGF23 promoter constitutes a proximal CRM anchored by the adjacent ETS1 and VDRE sites. To test this hypothesis, we dissected the mouse FGF23 promoter kindly supplied by Drs Mikiko Ito and Ken-ichi Miyamoto of the Tokushima University (Ito et al. 2005). By employing progressively truncated promoter-reporter constructs, we obtained evidence that a VDRE is located somewhere between -200 and $-400 \mathrm{bp}$ in the mouse FGF23 promoter (Fig. 8A and B). The activity of the FGF23 promoter fragments was unaffected by phosphate concentration (Fig. $8 \mathrm{~A}$ vs B), suggesting that phosphate-responsive elements must reside outside of the $1.0 \mathrm{~kb}$ promoter. To determine if the VDRE localized to the -200 to -400 interval corresponds to the newly revealed candidate VDRE shown in Fig. 7, this VDRE/ Nurr1 and/or its adjacent ETS1 site were inactivated by point mutation within the context of a $-0.6 \mathrm{~kb}$ promoter

Published by Bioscientifica Ltd 
A

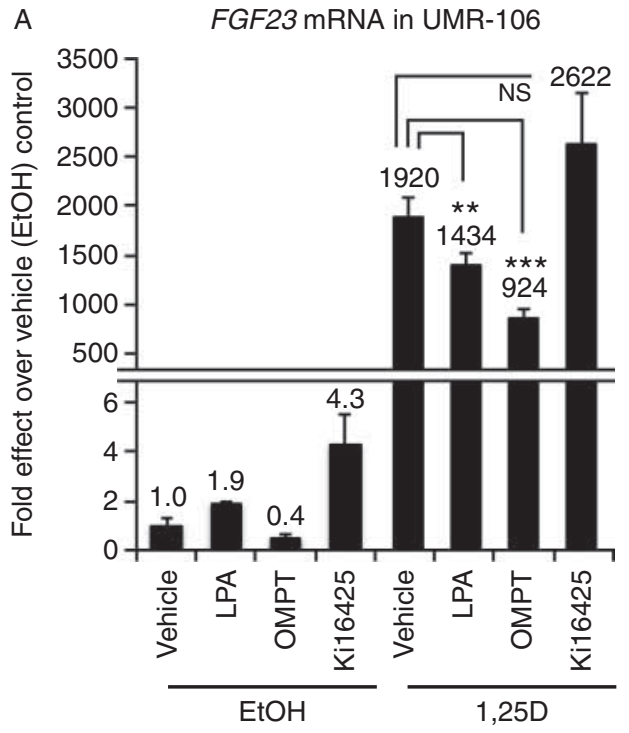

B

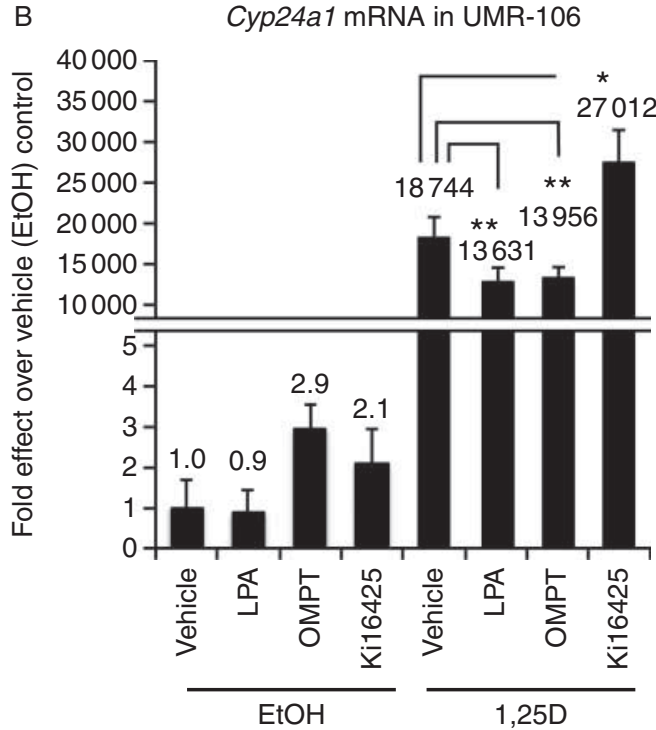

Figure 5

Induction of FGF23 and Cyp24a1 mRNAs by 1,25D in UMR-106 rat osteoblast/osteocyte-like cells and its modulation by lysophosphatidic acid (LPA). UMR-106 cells were treated for $24 \mathrm{~h}$ with $20 \mu \mathrm{M}$ LPA, $10 \mu \mathrm{M}$ OMPT

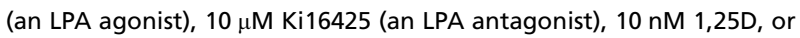
combinations thereof as indicated. mRNA levels were determined by real-time PCR for the following genes: (A) FGF23 and (B) Cyp24a1. Each bar represents the average of three independent experiments performed in triplicate \pm s.D. All statistics are calculated relative to the EtOH control, set at 1.0. ${ }^{*} P<0.05, * * P<0.01$, and $* * * P<0.001$.

fragment-luciferase construct and the mutated plasmids evaluated for $1,25 \mathrm{D}$ responsiveness. As illustrated in Fig. $8 \mathrm{C}$, the $-0.6 \mathrm{~kb}$ promoter fragment-luciferase construct yields a fourfold response to $1,25 \mathrm{D}$. This effect is significantly diminished to a 2.2- to 2.4-fold by the mutation of either the VDRE/Nurr1 or ETS1 site, or both

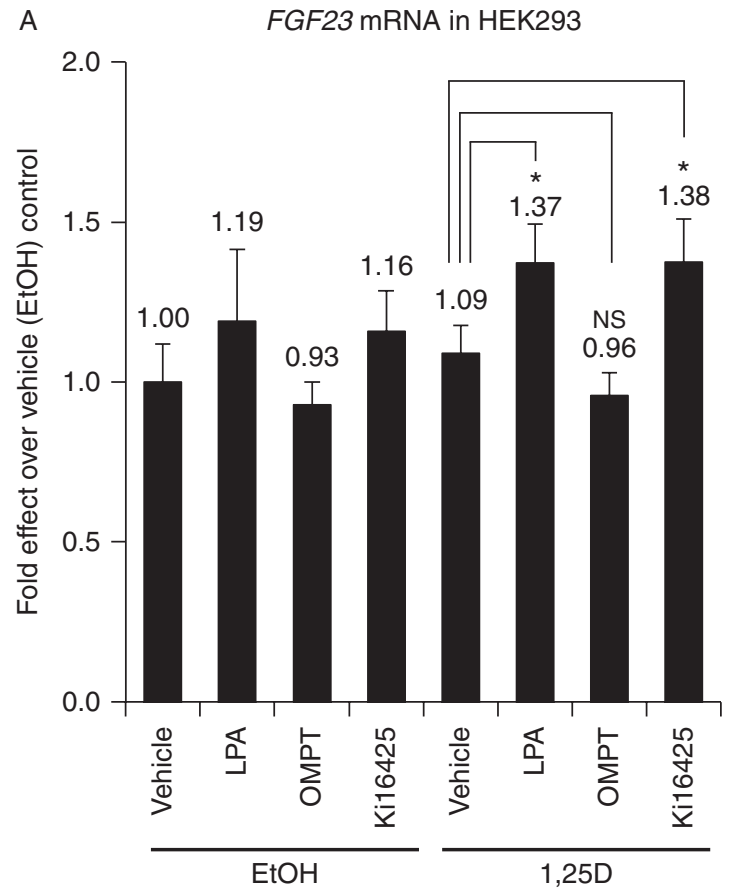

B

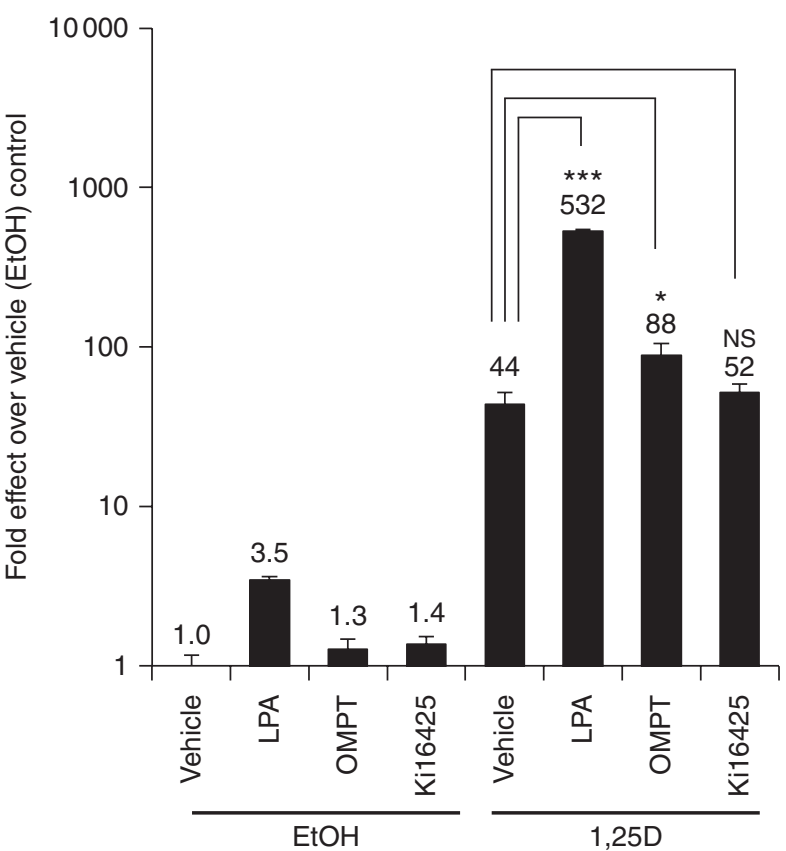

Figure 6

Control of FGF23 and CYP24A1 mRNAs by 1,25D in HEK293 embryonic human kidney cells and its modulation by lysophosphatidic acid (LPA) agonists/antagonists. HEK293 cells were treated for $24 \mathrm{~h}$ with $20 \mu \mathrm{M}$ LPA, $10 \mu \mathrm{M}$ OMPT (an LPA agonist), $10 \mu \mathrm{M}$ Ki16425 (an LPA antagonist), $10 \mathrm{nM}$ $1,25 \mathrm{D}$, or combinations thereof as indicated. mRNA levels were determined by real-time PCR for the following genes: (A) FGF23 and (B) CYP24A1. Each bar represents the average of three independent experiments performed in triplicate \pm s.D. All statistics are calculated relative to the $\mathrm{EtOH}$ control, set at 1.0. $* P<0.05$ and $* * * P<0.001$.

Published by Bioscientifica Ltd. 
VDREs: AGTGGGgacAGGTCA Sense GGAACTCagTAACCT Antisense ETS1 site GATA Site Lef1 site CRE/AP1 site Bold type= Nurr1 site

$-1158$

TGATGTCTATCTTGTGGGATACTCATCTGTTGTAGGAACTCagTAACCTGGGACACTGAGTGAGCAGTCCTTTT CTTGTGGCCATATACTGCTGATGTCTGTTCCAGCAGTCTGCCTTCCAATGGACTGTGTGTGTTAGTCTGCCTTC AACCTCCTACCCCACACACCACACCCCACACCCCACACCGCTGCATCAGACGCACCTCCACAGAGGGCATGCAC AGAGCAATTTCTGCAGTGAGGCATCCACTCATACAAGGTCCCAGGGTGTGGACATGGGCTGGGGAGGTGCCCAG TCCTGCAGCTCCTGGATGCTTCCTGCCCTCTGATTCCTCTGATCAGCCAGCAGTGCCGTTCCAGTCCTCCAAAT GAGTCTCCTTCCTATTGGCAAAGCCATAATTGCCAGTTTAGTTCCCTGCCTCATCCAGACGAGGGAAACTGAGA AACCAGATCTTGCCATTTTTGCTGACCTCAAGACCTTGTTTTCTTTCTTCTTGCCTTGAGGTCTTTGGGAATGG TGATGGGAGGTGTTGAGCTCATGCTCCCACCTAACACCCTTCCTGGGGTTTGTGTATGGGGGTAGGGGGGAGTC TCATTTGCCTGATAGCATCACTTATGACCATATATCAAGACACTTGCCAGATGCAACAGCCAGGAGTAAGCTCC AAGAACACACTTGGCAGCTGGAGGAAAAGGGCTTAAGCAAACCAAAACAAGGACACTGGAGGGAGATGAGTTAG CGAGGAGGCGGCTTTCTGGTTTTCTGGGGTTTTTTTTGTTTGTTTGTTTCAGTACTGCTGGCTGCCTTCACACT TCCTGATGGAAGTGGGوaCAGGTCAACAAATGACCCAGGGTCACAGATAACTTTTGCCCACACATCATTCACTT ATGGGAGCACTGGCTTGAAATTGAGGGGTGTGTGCGTGCATGTATGTGTGTGCCTGGAACTGACGCGCCTTCCG CAAGCCTAAGAAGTCTGGGCTTTTTCTTTGAATGGATGATTACAACACAGAGGATGTGGCGGCATTGTTTTTCC TGCTTGATGTCACACCACCACCCTTTAAAAGTCCCGGGGAAAAAAGGAGGGAATCTAGCCCAGGATCCCCACCT CAGTTCTCAGCTTCTTCCTAGGAAGAAGAGAAAGGCCAGCAAGGGCCCAGCCTGTCTGGGAGTGTCAGATTTCA AACTCAGCATTAGCCACTCAGTGCTGTGCAATG

\section{Figure 7}

Nucleotide sequence of the mouse FGF23 promoter. The color-coded legend for cis elements is depicted above the sequence. Nurr1 sites are depicted in bold type. The transcription start site begins at the $5^{\prime}$ end of the

simultaneously, with all responses by the mutated promoters not significantly different from the minimal promoter $(-0.06 \mathrm{~kb})$ control construct. The observation that the inactivation by site-directed mutagenesis of either the VDRE/Nurr1 or ETS1 elements abolishes transcriptional stimulation by $1,25 \mathrm{D}$ (Fig. 8C) provides evidence for a novel composite ETS1-VDRE/Nurr1 cis element that may be central to the CRM in the mouse FGF23 promoter that mediates at least part of the response of this gene to induction by $1,25 \mathrm{D}$. Finally, we probed the responsiveness of the FGF23 promoter to calcium, as David et al. (2013)
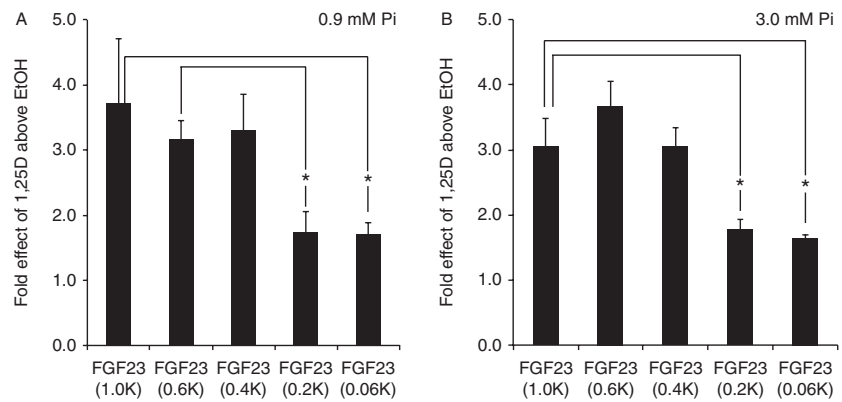

\section{Figure 8}

Dissection of the mouse FGF23 proximal promoter. (A) A promoter fragment containing $1.0 \mathrm{~kb}$ of promoter sequence $(1.0 \mathrm{~K})$ was linked to a luciferase reporter and transfected into K562 cells. Transcription of the reporter gene was stimulated three- to fourfold by 1,25D in the presence of $0.9 \mathrm{mM}$ (low) phosphate. This transcriptional effect is significantly reduced to less than twofold when progressively $5^{\prime}$-truncated promoter fragments (e.g., $0.4 \mathrm{~K}$ to $0.2 \mathrm{~K}$ ) are tested. (B) The profile of transcriptional stimulation by $1,25 \mathrm{D}$ of FGF23 promoter fragments, transfected into K562 cells, is essentially unaffected by the $3.0 \mathrm{mM}$ (high) phosphate concentration when compared underlined sequence. The TATA-box and the ATG start codon for translation are highlighted in light green. A full colour version of this figure is available at $h t t p: / / d x . d o i . o r g / 10.1530 / J O E-15-0225$.

recently called attention to calcium as a stimulator of FGF23 secretion by osteoblasts. Indeed, as illustrated in Fig. $8 \mathrm{D}$, high calcium significantly (1.7- to 2.0-fold) induces the $-1.0 \mathrm{~kb}$ mouse FGF23 promoter-reporter construct when it is transfected into MC3T3-E1 osteoblast-like cells. Therefore, as documented by data in Fig. $8 \mathrm{C}$ and $\mathrm{D}$, we conclude that $1,25 \mathrm{D}$ and calcium comprise FGF23 inducers that function by stimulating the proximal promoter of the mouse gene and likely other species, as the CRM components of this promoter are largely conserved across mammalian species.
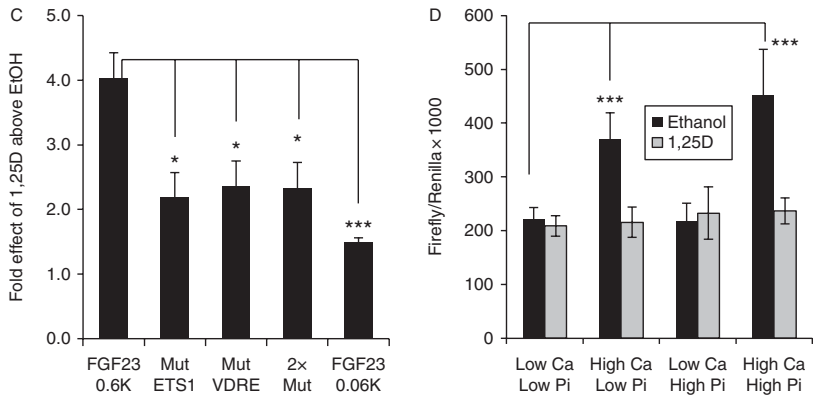

to the $0.9 \mathrm{mM}$ phosphate results. (C) When transfected into $\mathrm{K} 562$ cells, a $-0.6 \mathrm{~kb}$ promoter fragment-luciferase construct of the mouse FGF23 promoter yields a fourfold response to 1,25D. This effect is significantly diminished to 2.2- to 2.4-fold by the mutation of either the VDRE or ETS1 site, or both simultaneously, with all responses not significantly different from the minimal promoter $(-0.06 \mathrm{~kb})$ control construct. (D) High $(6.0 \mathrm{mM})$ calcium, but not high $(3.0 \mathrm{mM}$ ) phosphate, significantly (1.7- to 2.0 -fold) induces the $-1.0 \mathrm{~kb}$ mouse FGF23 promoter-reporter construct when it is transfected into MC3T3-E1 osteoblast-like cells. ${ }^{*} P<0.05$ and ${ }^{* * *} P<0.001$.

Published by Bioscientifica Ltd. 


\section{Discussion}

Like PTH, FGF23 inhibits renal Npt2a and Npt2c to elicit phosphaturia (Shimada et al. 2004). In contrast to PTH that is downregulated by $1,25 \mathrm{D}$ in parathyroid glands, FGF23 is upregulated by 1,25D in osteocytes (Kolek et al. 2005, Liu et al. 2006, Bergwitz \& Juppner 2010), which are a major source of endocrine FGF23 production by bone. Hyperphosphatemia enhances osteocytic FGF23 production independently of $1,25 \mathrm{D}$, rendering FGF23 the ideal phosphaturic counter-1,25D hormone because it inhibits renal phosphate reabsorption and 1,25D biosynthesis via repression of CYP27B1, while enhancing 1,25D degradation by inducing CYP24A1 in all tissues (Shimada et al. 2004). In this fashion, FGF23 allows osteocytes to communicate with the kidney to govern circulating $1,25 \mathrm{D}$ as well as phosphate levels, thereby preventing excess $1,25 \mathrm{D}$ function and hyperphosphatemia, either of which lead to ectopic calcification.

In the past year, new actions of FGF23 have been revealed via the generation of FGF23-null mice. For example, FGF23 deficiency leads to mixed hearing loss and middle ear malformation in mice (Lysaght et al. 2014). FGF23 also regulates renal sodium handling and blood pressure (Andrukhova et al. 2014a), as well as cardiac structure and function (Agarwal et al. 2014). Additionally, FGF23 is a negative regulator of prenatal and postnatal erythropoiesis (Coe et al. 2014) and a stimulator of renal calcium reabsorption through the TRPV5 channel (Andrukhova et al. 2014b). Many, but not all, of these novel actions of FGF23 involve the kidney and the vascular system. The function of FGF23 in adipose is unknown, but in the present communication we report a surprising repression of FGF23 expression by 1,25D in adipocytes, contrasting with all previous reports of FGF23 induction by $1,25 \mathrm{D}$ in other cell types. The mechanism of this repression is also unknown and is apparently unique for 1,25D control of adipose-expressed genes, differing in direction and 1,25D dose response when compared with the regulation of genes such as $C / E B P \beta, P P A R \gamma$, resistin, $a P 2$, the leptin receptor, and Cyp24a1. The only similarity between the effect of 1,25D on FGF23 and another gene is that of leptin, a gene product that itself induces FGF23. Thus, the influence of 1,25D to repress adipose FGF23 may be related to curbing some deleterious off-target effect of FGF23 in the adipocyte, such as adipose growth (Oldknow et al. 2015) or malignancy (Jacobs et al. 2011). With respect to obesity, FGF23 has been implicated in the regulation of energy metabolism, and mouse models with elevated FGF23 display increased adiposity (Oldknow et al. 2015), suggesting that $1,25 \mathrm{D}$ attenuation of FGF23 may curtail obesity. We (Kolek et al. 2005) originally reported that, in osteocyte-like UMR-106 cells, FGF23 mRNA levels are dramatically upregulated by $1,25 \mathrm{D}$. We later determined that this FGF23 induction is potentiated by leptin and inhibited by interleukin 6 (Saini et al. 2013), further linking FGF23 homeostasis with adipose hormones and cytokines, possibly implicating FGF23 repression by $1,25 \mathrm{D}$ in the modulation of energy metabolism and the prevention of malignancy.

In the current study, we reexamined the mouse FGF23 proximal promoter for transactivator binding sites that might mediate control of FGF23 mRNA expression. Notably, ETS1, which was implicated as a partner with VDR in controlling FGF23 in a previous study (Saini et al. 2013), possesses a cis-docking site in the mouse FGF23 promoter that is only $6 \mathrm{bp} 5^{\prime}$ of a newly discovered candidate VDRE/Nurr1 site (AGTGGGgacAGGTCA, highlighted in light purple in Fig. 7). By employing progressively truncated promoter-reporter constructs, we deduced that a $1,25 \mathrm{D}$-responsive region must be present between -200 and -400 bp in the mouse FGF23 promoter (Fig. 8A and B). When two elements within this interval, namely the candidate VDRE/Nurr1 site at $-334 \mathrm{bp}$ and/or its adjacent ETS1 site at $-346 \mathrm{bp}$, were inactivated by point mutation, transcriptional activation was diminished to a level not significantly different from that of the minimal promoter $(-0.06 \mathrm{~kb})$ control construct (Fig. 8C). Therefore, it was concluded that the -200 to $-399 \mathrm{bp}$ region of the mouse FGF23 promoter contains a CRM anchored by the adjacent ETS1 and VDRE/Nurr1 sites.

With respect to the $1,25 \mathrm{D}$ induction of murine FGF23, there are other considerations for the $-399 \mathrm{bp}$ region of the FGF23 promoter (Fig. 7). In addition to the ETS1VDRE/Nurr1 composite element, there exist two additional ETS1 sites that are conserved across species, as well as two conserved GATA cis elements of unknown significance (Barthel et al. 2007). The ETS1 protein is known to complex with VDR (Dittmer 2003) and to confer ligand- and AF2-independent transcriptional activation properties on nuclear receptors such as VDR (Tolon et al. 2000). The multiple conserved ETS1 cis elements in the FGF23 gene region (Saini et al. 2013) therefore render this transcription factor a likely candidate for the recruitment of VDR for the purpose of regulating FGF23 gene expression. Finally, the ETS1 protein is induced by $1,25 \mathrm{D}$ to amplify the response (Saini et al. 2013), and this process would be inhibited by cycloheximide, perhaps accounting for the reported cycloheximide sensitivity of the induction of FGF23 by 1,25D in UMR-106 osteocyte-like cells.

Published by Bioscientifica Ltd. 
Equally interesting is the existence in the FGF23 of a Lef1 site (Fig. 7), a cis element that is often associated with VDREs in 1,25D-regulated genes (Luderer et al. 2011). This Lef1 site is yet another target for 1,25D, possibly secondary to the repression of sclerostin (St John et al. 2014), a Wnt signaling inhibitor that is crucial in bone remodeling. Moreover, PTH increases FGF23 mRNA levels, and this effect is mimicked by the protein kinase A (PKA) activator, forskolin. PTH also decreases SOST mRNA encoding sclerostin, which is a PTH receptor (PTH1R) target; this action of PTH was abrogated by added sclerostin. Therefore, PTH increases FGF23 through the PKA and Wnt pathways (Lavi-Moshayoff et al. 2010). Thus, by analogy with PTH action on FGF23 expression, we suspect that the response of FGF23 expression to $1,25 \mathrm{D} / \mathrm{VDR}$ may not be a direct one, primarily because VDR has never been observed to associate with putative VDREs in the FGF23 gene region as monitored by ChIP-Seq analysis (St John et al. 2014).

Furthermore, control of FGF23 transcription by PTH has recently been discovered to be dependent on the induction of Nurr1 by PTH/PKA signaling (Meir et al. 2014). Conserved Nurr1 elements have been reported (Meir et al. 2014) in the FGF23 proximal promoter and immediately preceding the start site for transcription (as highlighted in bold type in Fig. 7). Although there is a conserved CREB/AP1 site just 11 bp upstream of the TATAbox in the rodent FGF23 genes (see Fig. 7), Meir et al. (2014) demonstrate that the effect of PTH via PKA/cAMP is apparently through induction of Nurr1, although it remains possible that the Nurr1 site just $3^{\prime}$ of the TATA-box mediates cooperation between Nurr1 and CREB to activate transcription. However, Meir et al. did not point mutate either of the Nurr1 sites in their study to demonstrate conclusively that one or both Nurr1 elements participated in the FGF23 regulation by PTH (Lanske \& Razzaque 2014). In the present experiments, our mutagenesis of the candidate VDRE at $-334 \mathrm{bp}$ in the mouse FGF23 promoter also would inactivate the Nurr1 site. This maneuver eliminated the response of the promoter to 1,25D (Fig. 7C), but one explanation of this result is that $1,25 \mathrm{D} / \mathrm{VDR}$ is functioning through Nurr1 as a secondary transcriptional activation factor as does PTH. We favor the mechanism that $1,25 \mathrm{D}$ induces FGF23 via the primary activation of Nurr1 expression rather than direct binding of VDR to the FGF23 promoter. Nurr1 then would bind to its cognate element adjacent to the ETS1 element and likely cooperate with ETS1 to induce FGF23. Evidence for this cooperation includes elimination of the 1,25D effect on FGF23 induction by point inactivation of the ETS1 site in question (Fig. 7C). Nurr1 has, in fact, been shown to interact with ETS1 in controlling the matrix metalloproteinase 1 promoter in cartilage, but in this case the relationship between the two transcription factors is an antagonistic one that does not require a Nurr1 consensus element (Mix et al. 2007). We do not know if the more proximal Nurr1 site $3^{\prime}$ of the TATA-box is relevant to the (secondary) action of $1,25 \mathrm{D}$ but point out that when cells are treated with $1,25 \mathrm{D}$, a significant 1.5 - to 2.0 -fold increase in reporter expression persists in $-0.06 \mathrm{~kb}$ truncated promoter-reporter constructs (Fig. 7A, B and C) that retain the proximal Nurr1 site. This residual effect may indicate that the proximal Nurr1 site is indeed active. But, more importantly, this new model of $1,25 \mathrm{D}$ action is consistent with evidence currently published on the 1,25D induction of FGF23 as follows: i) the secondary mechanism accounts for the cycloheximide sensitivity of the process (Haussler et al. 2010), ii) invoking Nurr1 is in concert with negative ChIP-Seq results for 1,25D-dependent association of VDR-RXR with putative VDREs in the FGF23 gene region in osteocytes (St John et al. 2014), iii) the secondary mechanism invoking Nurr1 is entirely consistent with a diminution of the 1,25D effect on the promoter when a Nurr1 site is altered by mutagenesis, and iv) Nurr1 expression is reduced in rat brain by developmental vitamin D deficiency (Kesby et al. 2013). Moreover, the requirement for a cAMP increase to induce Nurr1 and, secondarily, FGF23 in the case of PTH induction of FGF23, is likely pertinent to the control of FGF23 by $1,25 \mathrm{D}$. Notably, we have demonstrated in the present results (Fig. 5A) that LPA, a known activator of $\mathrm{G}_{\mathrm{i}}$ to suppress cAMP (Mansell \& Blackburn 2013), attenuates the ability of $1,25 \mathrm{D}$ to induce FGF23 in UMR-106 cells. Thus, we conclude that Nurr1, in combination with upstream PKA signaling, may be the new key to understanding FGF23 regulation by the calcemic hormones PTH and 1,25D. Indeed, one of the presentations of McCune-Albright syndrome, in which there is constitutive activation of $\mathrm{G}_{\mathrm{s}}$, is hypophosphatemia and FGF23 excess (Dumitrescu \& Collins 2008). Vitamin D has been associated with the development of the dopaminergic nervous system (Harms et al. 2011), a phenomenon dependent on Nurr1 (Sakurada et al. 1999). Therefore, another connection between Nurr1 and $1,25 \mathrm{D}$ action could reside in the CNS. Thus, a more complete characterization of the relationship between 1,25D and FGF23 could potentially open new vistas far beyond the treatment of bone mineral disorders to include not only energy metabolism and malignancy but also the prevention of neuropsychiatric disease.

Published by Bioscientifica Ltd. 


\section{Declaration of interest}

The authors declare that there is no conflict of interest that could be perceived as prejudicing the impartiality of the research reported.

\section{Funding}

This study was supported by National Institutes of Health grants NIH DK033351 to M R H and CA140285 to P W J.

\section{Author contribution statement}

Conception and design: I K, R K S, G K W, P W J, and M R H. Development of methodology: P W J, G K W, I K, R K S, and K P G. Acquisition of data: R K S, G K W, K P G, and I K. Writing, review, and/or revision of the manuscript: $M R H, G K W, P W J, I K$, and R K S. Administrative, technical, or material support: G K W, P W J, and M R H. Study supervision: P W J, G K W, and $\mathrm{M} \mathrm{RH}$.

\section{Acknowledgements}

The authors are grateful to Drs Mikiko Ito and Ken-ichi Miyamoto of the Tokushima University for the kind gift of mouse FGF23 promoter-reporter constructs.

\section{References}

Agarwal I, Ide N, Ix JH, Kestenbaum B, Lanske B, Schiller NB, Whooley MA \& Mukamal KJ 2014 Fibroblast growth factor-23 and cardiac structure and function. Journal of the American Heart Association 3 e000584. (doi:10.1161/JAHA.113.000584)

Andrukhova O, Slavic S, Smorodchenko A, Zeitz U, Shalhoub V, Lanske B, Pohl EE \& Erben RG 2014a FGF23 regulates renal sodium handling and blood pressure. EMBO Molecular Medicine 6 744-759. (doi:10.1002/ emmm.201303716)

Andrukhova O, Smorodchenko A, Egerbacher M, Streicher C, Zeitz U, Goetz R, Shalhoub V, Mohammadi M, Pohl EE, Lanske B et al. 2014b FGF23 promotes renal calcium reabsorption through the TRPV5 channel. EMBO Journal 33 229-246. (doi:10.1002/embj.201284188)

Barthel TK, Mathern DR, Whitfield GK, Haussler CA, Hopper HA IV, Hsieh JC, Slater SA, Hsieh G, Kaczmarska M, Jurutka PW et al. 2007

1,25-Dihydroxyvitamin D3/VDR-mediated induction of FGF23 as well as transcriptional control of other bone anabolic and catabolic genes that orchestrate the regulation of phosphate and calcium mineral metabolism. Journal of Steroid Biochemistry and Molecular Biology 103 381-388. (doi:10.1016/j.jsbmb.2006.12.054)

Bergwitz C \& Juppner H 2010 Regulation of phosphate homeostasis by PTH, vitamin D, and FGF23. Annual Review of Medicine 61 91-104. (doi:10.1146/annurev.med.051308.111339)

Coe LM, Madathil SV, Casu C, Lanske B, Rivella S \& Sitara D 2014 FGF-23 is a negative regulator of prenatal and postnatal erythropoiesis. Journal of Biological Chemistry 289 9795-9810. (doi:10.1074/jbc.M113.527150)

David V, Dai B, Martin A, Huang J, Han X \& Quarles LD 2013 Calcium regulates FGF-23 expression in bone. Endocrinology 154 4469-4482. (doi:10.1210/en.2013-1627)

Dittmer J 2003 The biology of the Ets1 proto-oncogene. Molecular Cancer 2 29. (doi:10.1186/1476-4598-2-29)

Dumitrescu CE \& Collins MT 2008 McCune-Albright syndrome. Orphanet Journal of Rare Diseases 3 12. (doi:10.1186/1750-1172-3-12)

Gidley J, Openshaw S, Pring ET, Sale S \& Mansell JP 2006 Lysophosphatidic acid cooperates with $1 \alpha, 25(\mathrm{OH}) 2 \mathrm{D} 3$ in stimulating human MG63 osteoblast maturation. Prostaglandins \& Other Lipid Mediators 80 46-61. (doi:10.1016/j.prostaglandins.2006.04.001)
Harms LR, Burne TH, Eyles DW \& McGrath JJ 2011 Vitamin D and the brain. Best Practice \& Research. Clinical Endocrinology \& Metabolism 25 657-669. (doi:10.1016/j.beem.2011.05.009)

Haussler MR, Haussler CA, Whitfield GK, Hsieh JC, Thompson PD, Barthel TK, Bartik L, Egan JB, Wu Y, Kubicek JL et al. 2010 The nuclear vitamin $\mathrm{D}$ receptor controls the expression of genes encoding factors which feed the "Fountain of Youth" to mediate healthful aging. Journal of Steroid Biochemistry and Molecular Biology 121 88-97. (doi:10.1016/j.jsbmb.2010.03.019)

Haussler MR, Whitfield GK, Kaneko I, Forster R, Saini R, Hsieh JC, Haussler CA \& Jurutka PW 2012 The role of vitamin D in the FGF23, klotho, and phosphate bone-kidney endocrine axis. Reviews in Endocrine \& Metabolic Disorders 13 57-69. (doi:10.1007/s11154-011-9199-8)

Haussler MR, Whitfield GK, Kaneko I, Haussler CA, Hsieh D, Hsieh JC \& Jurutka PW 2013 Molecular mechanisms of vitamin D action. Calcified Tissue International 92 77-98. (doi:10.1007/s00223-012-9619-0)

Ito M, Sakai Y, Furumoto M, Segawa H, Haito S, Yamanaka S, Nakamura R, Kuwahata M \& Miyamoto K 2005 Vitamin D and phosphate regulate fibroblast growth factor-23 in K-562 cells. American Journal of Physiology. Endocrinology and Metabolism 288 E1101-E1109. (doi:10.1152/ajpendo. 00502.2004)

Jacobs E, Martinez ME, Buckmeier J, Lance P, May M \& Jurutka P 2011 Circulating fibroblast growth factor-23 is associated with increased risk for metachronous colorectal adenoma. Journal of Carcinogenesis 103. (doi:10.4103/1477-3163.76723)

Jurutka PW, Hsieh JC, Remus LS, Whitfield GK, Thompson PD, Haussler CA, Blanco JC, Ozato K \& Haussler MR 1997 Mutations in the 1,25dihydroxyvitamin D3 receptor identifying C-terminal amino acids required for transcriptional activation that are functionally dissociated from hormone binding, heterodimeric DNA binding, and interaction with basal transcription factor IIB, in vitro. Journal of Biological Chemistry 272 14592-14599. (doi:10.1074/jbc.272.23.14592)

Kesby JP, Cui X, Burne TH \& Eyles DW 2013 Altered dopamine ontogeny in the developmentally vitamin $\mathrm{D}$ deficient rat and its relevance to schizophrenia. Frontiers in Cellular Neuroscience 7 111. (doi:10.3389/ fncel.2013.00111)

Kolek OI, Hines ER, Jones MD, Lesueur LK, Lipko MA, Kiela PR, Collins JF, Haussler MR \& Ghishan FK 2005 1\{a\},25-Dihydroxyvitamin D3 upregulates FGF23 gene expression in bone: the final link in a renal-gastrointestinal-skeletal axis that controls phosphate transport. American Journal of Physiology. Gastrointestinal and Liver Physiology 289 G1036-G1042. (doi:10.1152/ajpgi.00243.2005)

Lanske B \& Razzaque MS 2014 Molecular interactions of FGF23 and PTH in phosphate regulation. Kidney International 86 1072-1074. (doi:10.1038/ ki.2014.316)

Lavi-Moshayoff V, Wasserman G, Meir T, Silver J \& Naveh-Many T 2010 PTH increases FGF23 gene expression and mediates the high-FGF23 levels of experimental kidney failure: a bone parathyroid feedback loop. American Journal of Physiology. Renal Physiology 299 F882-F889. (doi:10.1152/ajprenal.00360.2010)

Liu S, Tang W, Zhou J, Stubbs JR, Luo Q, Pi M \& Quarles LD 2006 Fibroblast growth factor 23 is a counter-regulatory phosphaturic hormone for vitamin D. Journal of the American Society of Nephrology 17 1305-1315. (doi:10.1681/ASN.2005111185)

Luderer HF, Gori F \& Demay MB 2011 Lymphoid enhancer-binding factor-1 (LEF1) interacts with the DNA-binding domain of the vitamin D receptor. Journal of Biological Chemistry 286 18444-18451. (doi:10.1074/jbc.M110.188219)

Lysaght AC, Yuan Q, Fan Y, Kalwani N, Caruso P, Cunnane M, Lanske B \& Stankovic KM 2014 FGF23 deficiency leads to mixed hearing loss and middle ear malformation in mice. PLOS ONE 9 e107681. (doi:10.1371/ journal.pone.0107681)

Mansell JP \& Blackburn J 2013 Lysophosphatidic acid, human osteoblast formation, maturation and the role of 1 $\alpha, 25$-dihydroxyvitamin D3 (calcitriol). Biochimica et Biophysica Acta 1831 105-108. (doi:10.1016/ j.bbalip.2012.04.005) 
Meir T, Durlacher K, Pan Z, Amir G, Richards WG, Silver J \& Naveh-Many T 2014 Parathyroid hormone activates the orphan nuclear receptor Nurr1 to induce FGF23 transcription. Kidney International 86 1106-1115. (doi:10.1038/ki.2014.215)

Meyer MB, Goetsch PD \& Pike JW 2010 Genome-wide analysis of the VDR/RXR cistrome in osteoblast cells provides new mechanistic insight into the actions of the vitamin D hormone. Journal of Steroid Biochemistry and Molecular Biology 121 136-141. (doi:10.1016/j.jsbmb. 2010.02.011)

Mix KS, Attur MG, Al-Mussawir H, Abramson SB, Brinckerhoff CE \& Murphy EP 2007 Transcriptional repression of matrix metalloproteinase gene expression by the orphan nuclear receptor NURR1 in cartilage. Journal of Biological Chemistry 282 9492-9504. (doi:10.1074/ jbc.M608327200)

Oldknow KJ, MacRae VE \& Farquharson C 2015 Endocrine role of bone: recent and emerging perspectives beyond osteocalcin. Journal of Endocrinology 225 R1-R19. (doi:10.1530/JOE-14-0584)

Ozono K, Liao J, Kerner SA, Scott RA \& Pike JW 1990 The vitamin Dresponsive element in the human osteocalcin gene: association with a nuclear proto-oncogene enhancer. Journal of Biological Chemistry 265 21881-21888.

Quarles LD 2012 Skeletal secretion of FGF-23 regulates phosphate and vitamin D metabolism. Nature Reviews. Endocrinology 8 276-286. (doi:10.1038/nrendo.2011.218)

Saini RK, Kaneko I, Jurutka PW, Forster R, Hsieh A, Hsieh JC, Haussler MR \& Whitfield GK 2013 1,25-Dihydroxyvitamin D(3) regulation of fibroblast growth factor-23 expression in bone cells: evidence for primary and secondary mechanisms modulated by leptin and interleukin- 6 . Calcified Tissue International 92 339-353. (doi:10.1007/s00223012-9683-5)

Sakurada K, Ohshima-Sakurada M, Palmer TD \& Gage FH 1999 Nurr1, an orphan nuclear receptor, is a transcriptional activator of endogenous tyrosine hydroxylase in neural progenitor cells derived from the adult brain. Development 126 4017-4026.

Shimada T, Hasegawa H, Yamazaki Y, Muto T, Hino R, Takeuchi Y, Fujita T, Nakahara K, Fukumoto S \& Yamashita T 2004 FGF-23 is a potent regulator of vitamin D metabolism and phosphate homeostasis. Journal of Bone and Mineral Research 19 429-435. (doi:10.1359/JBMR. 0301264)

St John HC, Bishop KA, Meyer MB, Benkusky NA, Leng N, Kendziorski C, Bonewald LF \& Pike JW 2014 The osteoblast to osteocyte transition: epigenetic changes and response to the vitamin D3 hormone. Molecular Endocrinology 28 1150-1165. (doi:10.1210/me. 2014-1091)

Tolon RM, Castillo AI, Jimenez-Lara AM \& Aranda A 2000 Association with Ets-1 causes ligand- and AF2-independent activation of nuclear receptors. Molecular and Cellular Biology 20 8793-8802. (doi:10.1128/ MCB.20.23.8793-8802.2000)

Yu X, Sabbagh Y, Davis SI, Demay MB \& White KE 2005 Genetic dissection of phosphate- and vitamin D-mediated regulation of circulating Fgf23 concentrations. Bone 36 971-977. (doi:10.1016/j. bone.2005.03.002)

Received in final form 29 June 2015

Accepted 6 July 2015

Accepted Preprint published online 6 July 2015
(C) 2015 Society for Endocrinology Printed in Great Britain 